\title{
Genetic Approaches to the Study of Aging
}

\author{
Richard A. Miller, $M D, P h D^{1}$
}

Can mouse genetics teach us enough about the biology of aging to guide the search for anti-aging medicines that can delay late-life illness? Recent progress gives reason for optimism, with new data showing that changes in single genes can extend average and maximal life span by $40 \%$. Mice with these genetic variants remain healthy, active, and cognitively intact at average ages that correspond to $110-120$ years of human life span. Multiple lines of evidence now point to a hormone, IGF-I, as a key influence on life span, with low IGF-I levels associated with extended longevity in multiple model systems. The goal of this research is not gene therapy - we have no idea of what genes to change, how to change them, or what harm such changes might do-but instead to use insights from the cell biology and endocrinology of genetically long-lived mice and other species to help develop drugs that manipulate aging and thus postpone the many diseases and disabilities that are typically troublesome in old age. The complete conquest of cancer or heart disease would each lead to an increase of a mere $\sim 3 \%$ in mean life span in humans, i.e. about a tenth of what can be accomplished, today, in laboratory animals of delayed aging. In this context the paltry commitment to research in biological gerontology (six cents per $\$ 100$ of NIH funding, for example) seems worth reconsideration. J Am Geriatr Soc 53:S284-S286, 2005.

key words: longevity, genetics, mice, IGF-I, mutations

$\mathrm{T}$ he issue for me is what mouse geneticists can tell us about aging and what we could potentially, some day, do about it. Geneticists can take three approaches to aging. They can study variations in genes among individuals or groups to see whether they modify the aging process. They can study races or breeds or subpopulations like Labrador retriever dogs and Chihuahua dogs to see how they differ in aging, and they can evaluate rare mutations that influence the aging process. The experiments we do in our laboratory

${ }^{1}$ University of Michigan Department of Pathology Geriatrics Center Ann Arbor VA Medical Center Institute of Gerontology.

Corresponding Author: Richard A. Miller, MD, PhD University of Michigan School of Medicine 5316 CCGCB, Box 09401500 East Medical Center Drive Ann Arbor, MI 48109-0940, Phone: 734-936-2122, Fax 734-647-9749.

Email: millerr@umich.edu

DOI: $10.1111 / \mathrm{j} .1532-5415.2005 .53490 . \mathrm{x}$ start with a population we call UM-HET3 mice. Basically we have a family of 1,800 animals that are all brothers and sisters. Every animal of our population shares half of its genes with every other animal in the population. We can analyze the DNA and genes from every mouse. Then we measure how old each mouse gets, how quickly, what diseases it has, whether it has cognitive problems or cataracts, and we just match the two sets of information to try to find how many genes there are that influence these processes, what those genes do, and where they are located.

Some of these genes act early in life to make animals get old fast and others act only late in life to make old age a more severe event. ${ }^{1}$ There are genes that act early in life in young adulthood to change traits. But, in the same mice, there are other genes that affect the same traits and do it in a way that cannot be detected in young adults, and only becomes apparent when the mice are middle aged or older. These genes affect the same traits, but they act later in life. When we start to tease apart the genes that affect aging in humans, we need to think about this. We need to be able to test people at several ages during their life spans to see which genes are affecting them early on and which genes are affecting them later. We have found genetic differences in a very wide range of age-sensitive traits, including bones, eyes, hormones, weight, what they die of. Are there any genes in these mice that influence the age at which they die? The answer is yes. For instance, there are a pair of genes on chromosomes 2 and 16; if the mouse is lucky enough to get the good ones, it lives 173 days longer than mice unlucky enough to get the bad ones. This is a big effect, several times larger than the effect you would see in the human population if you cured cancer, heart disease, stroke, and diabetes. We then looked at mice that died of cancer because we wanted to see if these genes affect the age at which cancer occurred, and the answer is yes. ${ }^{2}$ We also looked at those mice in the population that died of something other than cancer. We wanted to know if the genes affect those diseases, too, and, again, the answer was yes with a similar-sized effect. So, our take home message was that there were genetic processes that influence the rate at which mice age, that is the likelihood of becoming a healthy old mouse, regardless of which diseases are likely to develop. This kind of evidence suggests that there might well be a specific aging process that we can learn enough about to modify or prevent.

The same kind of information allows us to investigate the idea that the rate of aging might be influenced by processes that start early in life; that turned out to be the case. 
Even as early as three months of age, we can measure something in these mice that tells us how likely it is that they will be in the long-lived group. It is easy; just weigh them. The smaller, shorter, light weight animals are the ones that live longer. ${ }^{3}$ The mice that are longer in their body length and heavier for that reason tend to die young. Something in the first few months of life influences the ultimate longevity of these animals.

What is it in the first few months of mouse life, or perhaps in the first few years of human life, that sets the stage to allow some people to be healthy in their 80 s, or to condemn others to be unhealthy and dead in the $60 \mathrm{~s}$ and 70 s? One of the factors is likely to be the hormone insulinlike growth factor-I (IGF-I). It is not insulin, but it is a hormone that resembles insulin and influences the rate of growth; in our population, those animals that have a lot of this insulin-like growth factor tend to die relatively young. Those animals that have a little of it are the small animals that tend to be long lived.

Dogs are a good example. ${ }^{4}$ If you go and get a St Bernard or a Wolfhound or a Great Dane, you are unlikely to have that companion ten or twelve years later. Big dogs tend to die young. Big dogs live, on average, about seven or eight years. Little dogs, like Chihuahuas and West Highland White terriers and Cairn terriers are not only light weight, but they are very long lived. In two studies in which it has been looked at, IGF-I appeared responsible for the difference between a breed of big dogs and a breed of little dogs. ${ }^{5,6}$ Lower levels of IGF-I were associated with small size and long life span as well. So, if it is true for dogs (and also for horses), and true for mice, it is likely, I think, to be true for people as well, that aging and life span are, in part, influenced by early life processes that modify body growth and body weight. We need to find out how nature does it and see if we can do it ourselves.

To get some additional insights, we compared laboratory and wild mice. The laboratory mice are big, fat, become sexually mature early, have big litters, but pay a price, namely shorter life spans. The wild mice are small, have small litters, take much longer to become sexually mature, and have 25 percent longer life spans. We think it is quite likely that the genes that are influencing life spans in our experiments are genes that actually are selected for their effects on reproduction, maturation, and growth, and that the aging consequence is just a side effect, but how that side effect works is what we now need to look at. We are mapping these genes because we can make crosses between the wild mice and the laboratory mice, we can see that the crosses come out right in the middle, which is good because we can now start to tease apart which genes are involved.

Rare mutants allow us to tease apart the aging process and the star mutant in our laboratory is the Snell dwarf mouse which stays one-third normal size throughout its entire adult life, and is very long lived. They live forty percent longer, and this is related to a change in a single gene. ${ }^{7}$ This gene lowers IGF-I and it also lowers thyroid hormone levels. This is consistent with the notion that changing these hormones early in life produces a mouse that may be small and less fertile, but has multiple advantages. As they get older, they look younger, have excellent cognition and learning, have excellent collagen, and resist cataract development. Interestingly, their cells are hard to kill in vitro with ultraviolet light, heating, herbicides, or trace metals. We think having cells that are hard to kill is a good thing for these mice. We think that's why they are so long lived, so cataract resistant, so cognitively intact, and we would like to use this system to find out what agents can cause cells in culture to become hard to kill, and then try to develop pharmaceutical agents for use in animals and humans. I would stress that these mice are not just alive longer, but they are healthy. That is, they have good joints, good lenses, good immunity, good cognition at ages at which the controls are already dead. And the hormones early in the life span, particularly IGF-I but also possibly thyroid hormones, seem to be a key part of that process. We think those are the causes, but the pathways connecting the hormones to the cell biology and then to disease resistance have not yet been defined.

Unfortunately, the things we can do in the laboratory to play around with longevity are trivial compared to what nature long ago figured out how to do. By caloric restriction, we can get about a fifty percent increase in longevity. That is not bad: it is better than you could do by conquering cancer, kidney disease, heart disease, stroke, etc. But, nature, even within a specific group of mammals, can do a lot better. The shortest lived rodents differ by a factor of 10 from the longest lived rodents. The shortest lived primates and the longest lived primates differ by a factor of about ten or so. If we want to make any real progress in this area, I think we need to figure out how nature does it. Closely related species, as we all know, age at significantly different rates. Baboons and people share about 97 percent of their genes, and of the three percent that are different, what we as gerontologists need to figure out is which tiny fraction of that three percent play a major role in the aging process to account for the fact that the baboon is likely to live about 20 years whereas humans live about 80 years. Many species can evolve a set of genes that slow down aging of the eyes, aging of the brain, of the immune system, aging of the muscles, everything that is associated with aging. We do not know whether nature always uses the same trick or not. Is it stress resistance or low free radicals or changes in hormones, better DNA repair, or other mechanisms or multiple mechanisms acting in concert? We need to find out how nature creates long-lived organisms.

Is there a common pathway used by caloric restriction and genetic mechanisms to increase longevity? The data suggest pretty strongly that, in some ways, caloric restriction mechanisms overlap, not always, but in some ways, with the dwarf mouse story. And that is interesting because that allows us to focus on the elements of the caloric restriction story that actually count, the ones that actually contribute to the longevity and the elements of the dwarf picture that actually contribute to the life span extension. From my point of view, what would be extraordinarily interesting is to know which ones are shared across species. Do long-lived dogs and bats, long-lived birds, naked mole rats, and porcupines share common physiological mechanisms that extend their life span?

Will genetic manipulation or genetic research help us to derive pharmacologic agents that allow people to maximize their physiological life span, with life expectancy at birth of 90 to 99 years? If we wear seat belts, drink just enough but not too much, and exercise a lot, eat a lot of vegetable 
foods, this is going to help us live longer and healthier lives, as will successful prevention or treatment of specific diseases. Could genetics help out? I think genetic models that have delayed aging like the dwarf mouse may point toward good preventive medicine if we find that, for example, in all of these models of aging, including those that are based on considerations of differences among species, blocking free radicals is the critical mechanism. That might focus even more attention on free radical blockers than currently exists. But if the genetic models point, instead, to DNA repair, or changes in hormone responses or something else, we'd need to direct our attention to those pathways rather than to free radical damage.

Will genetic manipulation or pharmacologic agents suggested by genetic research offer a realistic possibility of life expectancy at birth of 110 or 120 or more years? Twenty years ago, this was a science fiction question, but no more, and it is interesting to speculate based on current evidence. I believe the answer is yes. We know that we can do this in mice and in rats, we know that by changing a single gene or by a modest dietary alteration, we can create healthy, cognitively intact, vigorous animals that live forty percent longer. That does not prove we can do it in humans, and, of course, it does not tell us how we can do it in humans, but it is no longer irresponsible to suggest that it could be done. Indeed, we ought to figure out how to do it because it's a good thing to do. I do not believe genetic manipulation is part of the answer. I do not think there's any rational basis now other than science fiction for thinking that we are going to get that far by fixing people's genes. We do not know what genes they are, and we do not know whether fixing them would be a good or bad thing, and we certainly do not know how to do it. So, this is not a genetic change story. Rather, it is a matter of using genetic research to learn about how the cells work and how the body works, and then designing pharmacological agents that make use of our genetic information to slow aging down. I think that is a realistic possibility. How far are we from achieving this goal? The answer is again speculation. The answer I would give is we are closer to this than we are to eliminating cancer or heart attacks. Of the hundred dollars spent by our government on medical research, six cents are spent on biology of aging. I think that's a mistake because we're not going to cure cancer in the next twenty years, we're not going to cure heart attacks or strokes, but given adequate resources, and given adequate numbers of dedicated scientists working on this, we have a realistic possibility of learning how to do something about aging, not to cure it, but to slow it down in medically important ways. Nearly all deaths from cancer, strokes, heart attacks and many other important illnesses occur in old age, and thus progress on practical ways to retard aging is likely to bring with it major retardation of all these forms of illness. To make real progress on this, so that you will be living longer, requires wisdom, a commitment of substantial dollars for research, and, of course, as usually is the case, some luck. Our goal is to find ways to allow adults who are already healthy and productive in their $50 \mathrm{~s}$ and $60 \mathrm{~s}$ to remain so for another three or four decades instead of one or two decades.

\section{REFERENCES}

1. Jackson AU, Fornes A, Galecki A et al. Multiple-trait quantitative trait loci analysis using a large mouse sibship. Genetics 1999;151:785-795.

2. Miller RA, Chrisp C, Jackson AU et al. Coordinated genetic control of neoplastic and nonneoplastic diseases in mice. J Gerontol A Biol Sci Med Sci 2002;57:B3-B8.

3. Miller RA, Harper JM, Galecki A, Burke DT. Big mice die young: Early-life body weight predicts longevity in genetically heterogeneous mice. Aging Cell 2002;1:22-29.

4. Miller RA. Kleemeier Award Lecture: Are there genes for aging? J Gerontol Biol Sci 1999;54A:B297-B307.

5. Eigenmann JE, Patterson DF, Froesch ER. Body size parallels insulin-like growth factor I levels but not growth hormone secretory capacity. Acta Endocrinologica 1984;106(4):448-453.

6. Eigenmann JE, Amador A, Patterson DF. Insulin-like growth factor I levels in proportionate dogs, chondrodystrophic dogs and in giant dogs. Acta Endocrinologica 1988;118(1):105-108.

7. Flurkey K, Papaconstantinou J, Miller RA, Harrison DE. Lifespan extension and delayed immune and collagen aging in mutant mice with defects in growth hormone production. Proceedings of the National Academy of Sciences of the United States of America 2001;98:6736-6741. 